\title{
Transmission initiatique et conscience écologique dans les nouvelles de Ludovic Obiang
}

Fabiola OBAME, Université de Bretagne Occidentale

La forêt est labyrinthe, mais elle est aussi refuge. C'est au cœur de la nuit la plus obscure que le voyageur aperçoit au milieu de ce désert sauvage et touffu, aux ombres menaçantes, une petite lumière briller, lumière dont il ne sait si elle est fée, sorcière ou ogresse, image de l'hospitalité et de ses risques. Car c'est de ça dont il est question, entre l'arbre et l'homme, d'hospitalité.

Alain Montandon

\section{Introduction}

La forêt est un espace aux multiples facettes : séductrice, elle conduit l'humain à violer les interdits et à franchir le seuil de l'inconnu, pour s'enraciner dans un monde transcendantal ; mystérieuse, elle inspire la crainte par son insaisissabilité qui place l'humain face à ses limites. L'univers sylvestre est de ce fait un obscur objet de désir, car elle demeure dans l'inconscient un lieu qui à la fois fascine et effraie. Symbole de vie, la forêt est empreinte de significations multiples et ne se laisse pénétrer que par celui ou celle qui s'initie à ses mystères et sait voir en elle davantage qu'un motif ornemental. Riche des nombreux récits qui s'y sont déroulés, l'espace forestier est un lieu qui se fait porteur de l'histoire collective de ceux et celles dont le passé est inscrit dans la terre. En ce sens, la forêt devient un lieu de transmission où se racontent des récits restés confinés dans le secret et souvent réservés aux initié(e)s ; en d'autres termes, à ceux et celles qui savent regarder la forêt, l'écouter, et qui ne font pas que la traverser sans la voir. La forêt à ce niveau se fait espace de transmission car elle initie à une connaissance de l'espace. Elle nous apprend à poser un regard sur le monde environnant et à s'émerveiller pour aboutir à une connaissance de l'oükos (en grec « habitat » ou «maison»). L'écrivain(e) s'inscrit à ce niveau dans une pratique de la vie et une conscience écologique qui viendrait décentrer notre regard vers le monde extérieur.

Le nouvelliste gabonais Ludovic Obiang fait assurément de la forêt une zone atemporelle dans lesquels mythes, croyances et rumeurs se chevauchent. Ses récits sont des « indices qui permettent de dessiner en creux, à travers la trame de ses histoires, les contours chatoyants d'une envoûtante Afrique » (Tache, 250) située entre deux mondes - celui de l'humain et celui des esprits. Site de recueillement, ses abords inhospitaliers ne l'empêchent pas d'être le terreau d'un savoir : à son seuil, on expérimente la jouissance de découvrir l'impénétrable et un «Gabon en voie de disparition [...] à cause d'une érosion pas si 
naturelle » (262). Le choix de cet auteur de faire de la forêt l'espace de narrativité de ses récits n'est pas anodin. Obiang se réclame d'une initiation aux mystères de la forêt et chacune de ces œuvres atteste de cette filiation mystique. Entre la musique qui s'échappe de sa harpe sacrée, son initiation aux feuilles qui donnent la vie de l'Iboga et ses nombreuses allusions au monde qui se trouve derrière le village, c'est-à-dire au village, il se définit lui-même comme un conciliateur capable d'attiser la flamme d'amour perdue pour la terre. Sa volonté de nous placer face à cet espace nous invite à ce titre à sortir de cette rationalité qui nous empêche de nous ouvrir au monde. C'est peut-être la raison pour laquelle la nature qu'il nous présente est révélatrice d'un univers inconnu secoué par un dualisme opposant l'humain à la nature. Il est de fait particulièrement attentif à décrire cette forêt équatoriale qui pour lui est « matrice de la vie » et ne «saurait cacher sa réalité brutale et luxuriante » (264). Il décrit non seulement des scènes se situant dans la forêt, mais nous plonge aussi en son sein pour nous (ré-)apprendre à vivre au quotidien dans un dialogue continuel avec le bruissement des arbres. Sous sa plume, la forêt prend vie et se dote d'une âme.

La problématique de ce travail consistera dès lors à montrer comment la forêt parvient à se faire espace anthropologique. De quelle façon l'auteur réussit-il à faire de l'univers sylvestre un lieu sacré, une «partition musicale » (272) qui délivre un message sur des récits cachés? L'analyse portera sur quelques récits d'Obiang issus des recueils de nouvelles suivants : L'enfant des masques (1999), Et si les crocodiles pleuraient pour de vrai... (2006) et La tache bleue (2016). L'écocritique littéraire nous permettra d'effectuer une interprétation de ces œuvres à l'aune des interactions entre humain et nature.

\section{Transmission initiatique}

L'historien Alain Corbin, dans La douceur de l'ombre (2013), perçoit la forêt en tant que support dans lequel se matérialise les histoires des temps écoulés. C'est une énigme où clarté et ombre s'enchevêtrent pour faire émerger les inscriptions des récits de vies antérieures qui reposent dans cet espace mémoriel :

Se tenir au pied de l'arbre, l'observer impose de faire silence, oblige à penser à ce qui oppose l'éphémère et le durable, à se confronter à une temporalité qui n'est pas celle de l'homme. La distance entre son caractère immémorial et la mémoire de soi est immédiatement ressentie; ce qui incite à l'interroger comme si la pensée se faisait espoir de sens, «désir d'adhésion au monde $\gg(31)$.

Le monde végétal serait en ce sens un passeur pour qui sait le voir et établir le dialogue. Espace de transmission, c'est un lieu de pèlerinage qui permet de se découvrir en se projetant 
à travers le temps tel que le fait le double du narrateur de la nouvelle intitulée «Ce que chuchote la mangrove ». Celle-ci relate l'histoire d'Emane Nkombe qui, en voulant remercier ses frères de l'avoir invité à participer à une partie de pêche (en dépit de l'aversion générale qu'il leur inspirait), se retrouve perdu dans la forêt alors qu'il pistait un pigeon au chant singulier. Au cours de cette quête, il se trouve malencontreusement pris dans les arcanes d'une forêt qu'il fréquentait pourtant depuis qu'il était en âge d'aller y traquer les bêtes. Pour la première fois, le jeune homme s'aperçoit que sa connaissance de la forêt est superficielle. Son angoisse se fait grandissante au cœur de ce labyrinthe en croisant un «joufflu poupon » (97), mi-homme mi-monstre, qu'il surprend en pleine méditation dans la mangrove, à murmurer des paroles inaudibles. Si ses sentiments vont de l'angoisse à l'effarement en s'approchant de cette créature, c'est aussi parce qu'il trouve le poupon livrant son « funeste destin » (98) à la forêt, dialogue improbable pour le narrateur qui reste incrédule devant un tel échange. La communication avec le monde végétal étant logiquement pour lui impossible, Emane demeure sceptique quant au fait que l'on puisse déchiffrer la parole qui se dégage de ces frondaisons sinistres. Pourtant, c'est spontanément que la créature lui demande s'il est également venu écouter la mangrove, comme s'il s'agissait là d'un acte naturel :

- Tu viens aussi pour écouter la mangrove?

- La mangrove ? Pourquoi la mangrove ? Elle parle?

- Bien sûr qu'elle parle. Elle dit des choses intéressantes pour qui a le temps de l'écouter. Autrement, elle est un peu folle ; elle parle à la manière du vent qui agite ses feuilles. Il faut vraiment tendre l'oreille et faire le tri de ses paroles.

- Toi tu sais le faire?

- Oui, j'ai appris à le faire. Mais ça prend beaucoup de temps. Ce n'est pas à la mesure de l'homme. (97)

Emane, tout au long de ce dialogue, s'exprime uniquement en posant à son interlocuteur des questions qui soulignent son trouble et son ignorance, alors que les réponses assurées de la créature augurent de la non-initiation du jeune homme au langage de ce lieu. En profane, il ignore comment décrypter le silence de la forêt ; c'est une connaissance à laquelle il ne peut accéder dans son état actuel. La romancière Belinda Cannone explique à ce sujet dans S'émerveiller (2017) que ce n'est que par un «geste esthétique de la pensée » (28) que l'on peut atteindre cet état de relation-appartenance qui conduit à écouter le langage environnant. Il faut atteindre ce qu'elle nomme l'état de « hors-soi ${ }^{1} »$ qui conduit à un mouvement poétique ouvrant à une conscience d'une altérité humaine et non humaine. Dans le cas d'Emane, pour y parvenir, il lui faut donc un guide, qu'il trouve en la personne de ce poupon, pour l'initier à la voie qui mène aux mystères de la forêt. Par cet acte, le monde se dévoile à lui. Il comprend 
que l'origine de la haine que lui vouent ses frères est la répétition d'une haine ancestrale. Il apprend aussi qu'il est la réincarnation de ce poupon qui se révèle être en réalité son homonyme qui fut assassiné par des membres de sa fratrie des années plus tôt. Cette errance dans la forêt aura donc eu pour but de le mettre en garde pour éviter que l'histoire ne se répète et qu'il ne partage le destin funeste de celui dont il est la réincarnation. Présente pendant son assassinat, la forêt sait par exemple que c'est par jalousie qu'il fut tué. C'est dans cette « mangrove millénaire, témoin silencieux et fidèle de toutes les histoires » («Chuchote », 98) que l'âme de son homonyme resta bloquée sur Terre. C'est naturellement que cette mangrove devient celle qui «apaise et lui apporte la sérénité » (100). Elle se fait terre sacrée d'où jaillit le passé, c'est-à-dire une injustice vieille de plusieurs années, pour empêcher la répétition d'un fratricide. La forêt, en ce sens, est représentative de la vie sociale et détentrice d'un savoir salvateur qui vient des origines pour expliquer les tensions présentes dans le village. Le même constat peut se lire dans L'Anté-peuple (1983) de Sony Labou Tansi alors que «le chef » fait le récit de sa déchéance dans le maquis au personnage principal Dadju : «Puis j'ai réussi à sortir de la ville. Devant moi c'était la forêt: j'ai couru. Les arbres, tous les arbres me semblèrent des ancêtres. Ils m'ont ouvert les bras. Les oiseaux m'ont ouvert leurs chants » (183). On peut lire dans ce passage l'univers forestier présenté comme un espace cognitif et salvateur. En pénétrant dans la forêt, «le chef » a le même parcours qu'Emane Nkombe. Ils trouvent tous deux non seulement un asile qui les épargne de la mort, mais en plus ils font l'expérience d'une forêt chaleureuse dans laquelle ils trouvent l'hospitalité que le village et la ville ne parviennent à leur offrir. On peut en déduire par là que pour connaître la forêt et comprendre son langage, il est nécessaire de s'immerger en elle et de surmonter ce qu'elle a d'inquiétant.

Cette transmission du savoir en milieu sylvestre se lit aussi dans le récit «Derrière la haie des bananiers » quand celle qu'on surnomme la Rougeaude (en raison de son teint clair) découvre la vérité sur cet homme qui vient chaque année aux mêmes dates se recueillir sur la tombe de sa concubine. Accusé d'avoir tué sa compagne en sacrifice rituel pour accélérer son ascension sociale, il effectue des visites continuelles sur la tombe de la défunte qui intriguent et suscitent les spéculations au village. C'est en chercheuse de vérité et pour sortir le vrai de ces diatribes que la jeune fille s'en va épier cet homme devenu la source des ragots qui font de lui le meurtrier de sa compagne. À l'orée de ce bois, dissimulée par l'opacité de la forêt, la Rougeaude tend l'oreille pour démêler le vrai du faux. Mais la vérité que la fillette découvre est autre. Contre toute attente, ce qu'elle écoute dans cette forêt, c'est le récit d'un amoureux épris de chagrin. Cet homme qui se tient en face d'elle pleure son unique amour Angélique. 
Cette dernière se suicide après qu'il découvre qu'elle l'a trompé avec son ancien mari, qui est le père des jumeaux qu'elle attendait. La Rougeaude comprend, tapie dans l'ombre, que ce que l'on a longtemps cru être un meurtre passionnel, est un geste de désespoir, le désespoir d'Angélique qui s'est éventrée pour tenter de prouver son amour, en dépit de son infidélité, à cet homme qu'elle aime encore plus que ses enfants. Ainsi, pour expier sa faute et se débarrasser des enfants de la trahison, elle se donne la mort. La Rougeaude voit donc le masque des rumeurs tomber entre ces arbres, pour laisser place à une vérité encore plus dérangeante. Le tronc d'arbre qui la cache du malheureux est également l'agent intermédiaire grâce auquel la vérité lui parvient. La présence de La Rougeaude en ces lieux au moment de la confession lui permet de faire partie du cercle restreint de ceux et celles qui savent. Elle devient donc une initiée, une privilégiée à qui parvient la parole sélective de l'oükos. Tel que l'indique le proverbe fang ${ }^{2}$ placé en exergue de la nouvelle - « Tu dis que tu fais quelque chose dans la solitude, alors que la solitude même te voit » (29) - , la nature devient ce témoin silencieux qui révèle la vérité. Elle est cette solitude, ce regard à focalisation omnisciente qui se fait clarté dans l'obscurité de la forêt, pour ouvrir un passage à une réalité inattendue. Elle est aussi ce silence qui vient faire taire les rumeurs pour céder place au vrai. De ce point de vue, l'espace forestier s'oppose à l'espace humain car tandis que le premier fait accéder au vrai, le second est fait de paroles incertaines et troubles. Dans cette autre manière de lire le passé, la géographie se fait reflet de l'histoire. En se révélant être un espace de transmission de savoir, la forêt est également un espace de mémoire. Elle est porteuse de bouts d'histoires qui permettent à nos personnages de retrouver leur identité en ces lieux et d'y tisser un sentiment d'appartenance. Parcourir la forêt reviendrait alors à remonter le temps et à visualiser l'histoire inscrite dans chaque sillon de ces arbres qui fixent les souvenirs de toute une communauté. Cette dimension cognitive de la spatialité associe la forêt à un «lieu de mémoire $^{3} »:$ tel que le souligne Christian Jacob, « les savoirs constituent [...] une dimension centrale des lieux de mémoire, car la mémoire se transmet et s'actualise grâce à la reproduction et à la transmission de récits fondateurs, de savoir-faire, de manières de dire, d'une mythologie collective »(6). De ce point de vue, lorsque nous affirmons que la forêt est un lieu de mémoire, c'est parce que l'univers sylvestre véhicule un récit qui contrebalance le récit humain.

Par ailleurs, la couverture du recueil de nouvelles L'enfant des masques corrobore l'hypothèse qui fait de l'espace sylvestre un lieu de transmission. Ce jeune garçon à la peau noire qui apparaît en page de couverture semble regarder le monde à partir de l'espace créé 
par les ramures de l'arbre. Hormis sa tête, les membres inférieurs de son corps se confondent aux feuilles d'un vert si profond qu'il semble composer et prolonger son nouveau corps. Comme si cette barrière verdoyante devenait un camouflage pour accéder à un savoir secret, la fixité de son regard laisse penser qu'il observe ce qui se passe de l'autre côté du monde végétal. Pour l'enfant qui semble se métamorphoser progressivement en arbre, la forêt devient autant une terre de reconstruction que de réincarnation. D'ailleurs, quand on se penche sur la nouvelle éponyme, cette idée de reconstruction paraît centrale au parcours du jeune garçon chez les masques avec lesquels il séjourne de force à la suite d'un kidnapping. C'est dans ce milieu qu'il commence peu à peu à comprendre qu'il s'est réincarné pour expier une faute commise lors de sa vie précédente. Avec ces masques qui vivent « au sein des artères de forêt les plus touffues, les plus ronceuses » («Enfant», 42), il reconstitue les éléments de sa vie précédente et recouvre des fragments de sa mémoire envolée. Cet enfant comprend que sa vie antérieure d'adulte n'a été qu'une succession de mauvais choix qui cause sa propre mort et celle de sa bien-aimée. Après «le rite du bain » qui marque sa renaissance et son appartenance aux masques, son séjour dans la forêt trouve une explication :

Alors tout s'éclaire, je comprends tout du serment qui me lie. J'atteins à la révélation suprême, la confluence de tous mes pas, depuis mon enlèvement initial jusqu'au prochain déchirement. Tout s'éclaire, dans un flamboiement de mes sens. Je me prête au sacrifice, celui de ma propre aura, sans souci des conséquences, car mon cri lui survivra. (66)

Cet acte initiatique permettra, dit-il, «d'accéder à ce qui représente à mes yeux le summum de leur savoir : la réincarnation dans l'homme» (63). Avec Odabor, la passeuse des âmes mortelles, il comprend que son retour sur terre a été voulu pour qu'il sauve Ada (l'enfant unique du réincarné et, pour le jeune garçon, une amie d'enfance) d'un mal incurable qui l'a fait délirer avant de la plonger dans un profond coma. C'est d'ailleurs à la jeune femme qu'il écrit une lettre relatant son passage chez les masques et à qui il raconte les méandres incongrus de sa vie antérieure qui l'ont mené lui, jeune «ministre du Christ » à céder à une passion adultérine qui donna le jour à Ada. C'est aussi dans cette lettre qu'elle retrouve le mea culpa de son père qui préfère s'enfuir du séminaire en s'enrôlant dans l'armée, où il trouve une mort désirée après le décès en couches de celle qu'il aimait. Dans les profondeurs de la forêt se dévoile sa propre existence. Sorti d'une carapace dans laquelle il s'est longtemps dissimulé, il affronte ses erreurs. L'univers sylvestre, en l'initiant au mystère du monde, lui offre la possibilité de reconstruire une nouvelle vie. 


\section{Conscience écologique}

Aldo Léopold, pionnier en matière d'écologie aux États-Unis, développe une philosophie de l'écologie dans son anthologie La conscience écologique en 2013 (publiée à titre posthume et composée de quinze textes inédits de l'auteur regroupés par Jean-Claude Génot et Daniel Vallauri). Il préconise une nouvelle perception de la nature reposant sur une utilisation intelligente de l'environnement et sur la protection des richesses naturelles. Pour l'essentiel, ce qu'il postule comme des comportements respectueux de l'environnement appelle à adopter une éthique environnementale. Il part du constat que l'échelle de nos valeurs doit être remise en question et refondée pour infléchir sur la courbe des évènements chaotiques que les scientifiques prédisent car les changements environnementaux ne résultent pas uniquement de l'essor de la science et des techniques. Le combat serait alors à la fois à mener sur un ordre moraliste, collectif et individuel, car il passe par l'adoption d'une éthique environnementale. Lorsque l'on s'attarde sur les personnages mis en scène par Obiang, on constate que l'auteur a beaucoup travaillé sur cet aspect d'une conscience environnementale chez ses personnages. Ils sont tous animés par un attachement profond à l'oükos et par une volonté de se «reterritorialiser ${ }^{4}$ » à la nature. L'usage récurrent d'analogies substituant l'homme à l'animal dans la nouvelle «Et si les crocodiles pleuraient pour de vrai... » en est représentatif. On constate par exemple dans les phrases qui suivent une comparaison entre les comportements humains et ceux des animaux :

Ils se comportaient entre eux comme les crocodiles du fleuve. Unis par un intérêt commun, mais capables de s'entredéchirer à la moindre anicroche. (137)

C'est ainsi qu'un jour, alors que nous étions à patauger comme des jeunes hippopotames dans l'eau du fleuve. (143)

C'est ce qu'il s'efforçait de croire, en enfonçant son cou dans la terre comme une autruche... (148)

À travers ces citations, Obiang affirme clairement son désir de rapprocher l'humain des animaux, comme s'ils étaient pour lui des êtres semblables. Il accorde également une place importante à la figure de l'animal qui concourt à laisser penser que, sous sa plume, la frontière existant entre l'humain et le non-humain n'existe pas. C'est à cet égard que dans la nouvelle «Et si les crocodiles pleuraient pour de vrai... », «crocodiles», « hippopotames » et « autruche[s] » se voient comparés à l'humain en raison d'une similitude entre les deux entités qui se trouverait dans leur comportement. Cette similitude est davantage frappante en raison du fait que, dans ces exemples, ce ne sont pas les animaux qui reproduisent des traits humains, 
mais plutôt le contraire. L'animal devient un être à imiter, un autre soi-même auquel on peut s'identifier. Comme si cette nature était ce qui lui permettait d'expliquer son monde, l'auteur utilise l'imaginaire environnemental pour servir de référent aux situations qu'il décrit. Alors que ses comparaisons assimilent l'humain à la nature a contrario, il semble anthropomorphiser la biogée ${ }^{5}$. On lit cette dimension à travers la personnification de l'Arambo, cette rivière qui traverse chacune des nouvelles jusqu'à devenir personnage principal des intrigues. C'est à elle que Mbane va porter sa peine dans «Jérôme Békale devait mourir ». C'est également elle qui serpente dans les souvenirs d'enfance du narrateur du «Rêve en miettes » qui vit sa première idylle avec Meviane. C'est encore elle qui accueille le corps sans vie de la mère de Moïse dans «Pour que l'Arambo coule toujours » (l'Arambo est le nom attribué à un cours d'eau dans la ville de Libreville au Gabon). Lieu de mémoire, espace d'étude, site de rencontre, objet de lutte, l'Arambo suscite la passion auprès de ceux et celles qui viennent se laisser bercer par son chant : ce fleuve devient l'actant incontournable de ces récits et sa musicalité, un refrain entêtant. De la personnification à l'anthropomorphisation, l'auteur fait de cette rivière une métaphore obsédante dans son écriture, qui souligne par là son rapport à l'environnement naturel. On remarquera de fait que quand ce n'est pas l'Arambo qui raconte une histoire d'amour tragique, c'est une tourterelle qui chante son désir de chair humaine («Comment se quittent les tourterelles ») ou un crocodile ancien enfant soldat qui se repent sur ses actes passés («Et si les crocodiles pleuraient pour de vrais...»). Ces éléments de la nature se dotent d'une parole et de sentiments, à l'instar des humains, pour donner un tournant nouveau au récit historique.

Dans ce paysage, Obiang met aussi en scène des personnages qui sont porteurs d'une éthique écologique qu'ils partagent avec leur entourage et qui réenchante le monde dans lequel ils vivent. C'est le cas dans le havre de paix construit par Señor Francis Engomo, ancien mercenaire qui accueille les enfants et leur transmet sa passion pour les arbres en leur confiant la tâche d'entretenir l'arbre-jumeau planté à leur arrivé dans l'orphelinat. De plus, que dire de cette fillette qui chaque jour parcourt le tendre sentier de la fugue ? Ce sentier la conduit pendant un court moment dans cette clairière qui attend le son enchanteur s'échappant de sa flûte en bambou. Comme pour réparer ses erreurs, l'ancien mercenaire Señor Francis Engomo reconstruit le monde en y plantant des arbres et en transmettant cet attachement à l'arbre aux orphelins, tandis que la fillette montre que la musique peut permettre de s'unir au monde.

Dans la nouvelle «Pour que l'Arambo coule toujours », le prêtre tente ainsi de défendre la rivière d'un Moïse qui tente de la condamner en construisant un barrage : 
L'Arambo ne peut pas mourir, elle doit continuer de couler, de pleurer sous la voûte de bambous. [...] Vous n'entendez pas la plainte qu'elle murmure, contre vous, contre tous ceux qui veulent barrer son cours ? [...] Venez, asseyez-vous près de moi, juste à côté de l'eau...Allons, ne craignez pas de vous mouiller, elle n'est pas méchante avec les inconnus. Maintenant écoutez, elle va parler, elle va vous révéler son secret. (52-53)

L'invitation à écouter ses secrets reste toutefois vaine car Moïse affirme ne pas être un «poète » et ne pas avoir l'«oreille pour entendre ces choses-là » (52). Son rapport à l'Arambo est différent de celui du prêtre qui y voit le lieu de mémoire qui a vu mourir la mère de Moïse alors qu'elle lui donnait naissance. Ces différents personnages montrent de sorte que la voie du «vivre-ensemble ${ }^{6} »$ reste accessible pour qui sait ancrer les rhizomes de son identité dans la terre.

La forêt est un cadre sociologique qui laisse supposer que les racines de notre humanité et de toute connaissance sont à trouver au cœur des ténèbres sylvestres. Cette archive du vivant vient souligner le fait que l'unité entre l'être et le monde naturel est la condition pour accéder au savoir sylvestre tel qu'on peut le voir à l'analyse du personnage de Biang Mekè. Considérée comme folle, la jeune fille vivant en ermite au creux de la forêt préfère la proximité des buissons et des animaux à celle des humains. Effrayante, elle est crainte par les villageois et villageoises à cause de son comportement fantasque, ses éclats de rire soudains et ses propos inquiétants qui laissent croire qu'elle communique avec l'au-delà. Cependant, c'est cette « fadette des ravines sombres » (137) qui est la guérisseuse du village. L'efficacité de ses décoctions naturelles est telle qu' «on avait fini par la confondre avec ses feuilles, on avait fini par l'appeler Mekè ${ }^{7}$. En parlant d'elle on disait Mekè ou même Biang Mekè, et c'était l'assurance d'un traitement sûr » (129). Sa science est sans égale car il se dit dans le village qu'elle lui viendrait des génies de la forêt. Cet être de nature vivant dans un style de vie marginalisé a accès au savoir des plantes Ironiquement, l'écrivain fait de ce personnage marginal le dernier espoir que l'on consulte quand la médecine conventionnelle montre ses limites. Est-ce pour signifier que c'est parce qu'elle vit dans la nature que sa compréhension du monde parait différente, voire supérieure à celle des autres ?

Similairement, dans le recueil intitulé La tache bleue, la nouvelle «L'exilé des hautes futaies » ne manque pas non plus de faire de la nature un élément qui redonne vie. Alors qu'il était atteint d'un mal incurable depuis plusieurs mois, le narrateur raconte comment ses forces lui revinrent à l'issue d'un voyage mystique initié par son manguier. Pour goûter «une dernière fois la fraîcheur de son ombre »(158), c'est assoupi au pied du manguier qu'il chérissait le plus dans son jardin qu'il recouvrit la santé : 
Quand je repris conscience, convaincu d'avoir définitivement perdu la vie, je dus pourtant admettre que j'étais de nouveau chez moi, affalé dans le même fauteuil, alors que le manguier était redevenu lui-même, manguier-nain dont le feuillage touffu et arrondi frémissait sous la caresse vesprée. En revanche, aussi invraisemblable que cela pût paraître, je n'avais plus le moindre sentiment de faiblesse. Je me sentais revigoré, fort, costaud, capable de signer un nouveau bail avec la vie. Cette virée sur les cimes du délire m'avait-elle guéri, ou bien était-ce l'ataraxie du paradis ? En tous les cas, je n'avais plus connu cette sensation depuis belle lurette. (164-165)

Il revient non seulement revigoré, mais aussi plus informé de la cause de sa maladie qui n'était que le résultat des machinations de ses serviteurs dont il avait fait ses héritiers testamentaires. Cela voudrait-il dire qu'il faudrait, comme le titre de la nouvelle l'indique, s'exiler dans les hautes futaies pour découvrir le monde tel qu'il est réellement? Est-ce parce que, tel qu'il l'affirme, il «aime les arbres, tous les arbres sans exception, dès lors qu'ils plongent leurs racines profondes dans le sol afin de mieux se projeter vers le ciel » (153) que cette rémission lui est accordée ? Quoi qu'il en soit, le rapport d'Obiang à la nature est fort. La place accordée à l'environnement naturel montre non seulement son attachement à la Terre mais aussi la conscience écologique qui traverse ses récits. Celle-ci met en surbrillance l'ethos de cet écrivain pour qui, tel que le note Corbin pour faire état du rapport de l'humain à la nature, «le paysage ne se réduit pas à un spectacle. Le toucher, l'odorat, l'ouïe surtout, sont aussi concernés par la saisie de l'espace »(Homme, 9). C'est pourquoi les personnages de ces nouvelles semblent liés à l'espace forestier par d'obscurs secrets ou par un engagement écologique très poussé. Leur vie devient le reflet d'un vivre-ensemble avec la nature qui semble inviter le lectorat à se joindre à cette ultime réconciliation avec la biodiversité. Plus qu'un éveil à un sentiment de nature, l'auteur va plus loin en posant les termes d'un nouveau contrat naturel $^{8}$ qui reposerait sur un lien plus sensible à la Terre. Ces récits, en montrant la valeur mystique, historique et écologique de l'environnement naturel, font d'Obiang le porteparole d'un monde qu'il veut plus ouvert à une conscience écologique. Le fait que chacun de ces recueils entretienne un lien avec l'environnement naturel montre, en effet, que c'est une question incontournable dans son écriture. La prégnance de cette thématique environnementale traduit une volonté de faire découvrir la forêt gabonaise et ses mystères à son lectorat. Dans ces représentations, il écrit donc des récits où l'intrigue implique du personnage principal de retourner dans cette forêt où les trames narratives semblent toujours trouver un dénouement heureux. Ses récits, dans cette perspective, invitent à connaître l'espace forestier, non pas seulement comme un lieu de passage, mais comme un lieu de connaissance de soi et de savoir sur le monde. La géographie se distille dans chaque page et 
devient une répétition qui vient souligner les tensions environnementales du présent. Le choix du genre littéraire ne représente-t-il pas alors son projet d'écrire pour un monde au bord de l'épuisement? Si, tel que le note Denise Paré dans son article «Habitats, migrations, prédations » (2008), la nouvelle est un genre capable de «saisir le moment cruel de la décomposition et de la dissolution » (456) du monde, la prose d'Obiang s'inscrit tout autant dans cette lignée. Dans ses brèves fictions, on est tenté de croire qu'au final, il veut inciter le lecteur à un dénouement où il serait un protagoniste clé qui poserait les termes d'une reconstruction de la nature.

\section{Conclusion}

La forêt apparaît sous la plume d'Obiang comme un espace interstitiel dans lequel le monde matériel et le monde sensible se rencontrent. Espace de sociabilité, la forêt casse les frontières entre l'humain et le non-humain pour les rendre plus conciliants. En son sein, l'humain interagit avec les autres espèces vivantes : il acquiert autant un savoir écologique que des connaissances sur lui-même pour tisser une relation nouvelle au monde. Dans ce lieu de l'errance, les personnages se livrent à une quête du savoir via une initiation aux lieux qui fait aussi accéder à une science restreinte, ignorée de tous. Cet espace labyrinthique permet ainsi d'accéder à des savoirs historiques, culturels et même sociaux. En faisant taire les rumeurs qui s'élèvent des cités humaines, la forêt crée une opposition et une tension entre les espaces car c'est dans le silence de la forêt que la vérité se révèle.

Cet aspect savant de la forêt, beaucoup l'ont rêvé, idéalisé, goûté, fantasmé. Déjà dans les contes lus aux enfants, on le retrouve aux côtés de Hans et Gretel, du Petit Poucet, de Mowgli, du Petit Chaperon rouge, de Blanche-Neige. Hervé Bazin représente aussi cette dimension dans L'église verte (1981) lorsque Godion et sa fille Claire découvrent un homme, qui se veut sans identité, au milieu d'une église qu'il considère comme refuge. D'autres comme Kate Grenville en ont fait un lieu où l'on peut revisiter l'histoire des luttes territoriales et raciales entre aborigènes et exilés britanniques dans The Secret River (2005). Les films tels que la trilogie Le seigneur des anneaux n'en font pas non plus l'économie. Ces œuvres retracent le parcours des protagonistes dans cet espace de l'interdit qui ouvre un chemin vers l'autre et devient un paysage intérieur où l'on se découvre. Le monde sylvestre est en cela un lieu transgressif dans lequel s'opère la reconstruction parce que c'est un espace où des vies se tissent et se dénouent. Il apporte une vision différente à ce topos et en fait un lieu de savoir, de renaissance, de guérison. Il s'agit également d'une reconstruction car la forêt dévoile les masques pour ceux qui la regardent autrement que par sa valeur paysagère, à savoir par sa 
capacité à initier l'humain aux secrets du monde et au langage de l'écosystème pour qui est capable de se laisser émerveiller.

Finalement, par sa production littéraire, Obiang convie son lectorat à voir que le silence des arbres peut abriter des ténèbres salvatrices. Subtilement, il crée une tension entre l'espace forestier et l'espace urbain qui semble signifier que la vérité ne peut sortir que de ce lieu de confessionnal qu'est la forêt. En cela, il donne à ceux et celles qui cherchent la vérité de se reconnecter à la terre et de devenir des «jardiniers de la forêt» (Tache, 260). Les nouvelles d'Obiang ont justement cela d'intéressant qu'elles montrent l'intérêt de cette reconnexion à la Terre. Le retour à la nature auxquelles elles invitent, reposent sur une initiation à son langage, et la transmission à son savoir s'effectue dès lors que l'on arrive à se placer face à elle en tant qu'altérité et non comme un décor. C'est un point sur lequel ces récits reviennent souvent, montrant que l'adoption d'une conscience écologique dépend du rapport entretenu avec la forêt. La relation entretenue entre l'humain et l'environnement naturel est déterminante : elle conditionne les perceptions que l'on pourrait en avoir.

Sans adopter un ton moralisateur sur la préservation de l'environnement, l'auteur se fait écologiste pour montrer l'importance d'un vivre-ensemble avec la nature. De plus, il incite à poser un regard autre sur la nature par les jeux de style qu'il emploie et la valeur sacrale et mémorielle dont il revêt la forêt. Et pour cela, il use fortement de son expérience de musicien et de musicologue pour montrer que le monde est une «partition musicale » comme il l'affirme dans La tache bleue. Ses récits sont donc des promenades sonores grâce auxquelles l'on pourrait découvrir " pourquoi les personnes sagaces entretenaient les forêts et parlaient aux arbres comme à des vivants »(134). Obiang conduit son lectorat à venir découvrir ce qui se cache derrière les masques d'une forêt, et de quelles autres vérités elle est porteuse, une vérité alternative qui confronte les personnages avec une autre partie d'euxmêmes qu'ils ignoraient ou avec un passé resté tapi dans le silence des forêts.

\section{Bibliographie}

Bazin, Hervé. L'église verte. Paris : Seuil, 1981.

Cannone, Belinda. S'émerveiller. Paris : Stock, coll. «Essais-Documents », 2017.

Corbin, Alain. La douceur de l'ombre. L'arbre, source d'émotions, de l'Antiquité à nos jours. Paris : Flammarion, 2013.

---. L'homme dans le paysage. Paris : Textuel, 2001.

Jacob, Christian. Qu'est-ce qu'un lieu de savoir?. Marseille : Open Edition P, coll. 
«Encyclopédie Numérique », 2014.

Obiang, Ludovic. Et si les crocodiles pleuraient pour de vrai.... Paris : Ndzé, 2006.

---. La tache bleue. Guadalajara : PU du Nouveau Monde, 2016.

---. L'enfant des masques. Paris : Harmattan, 1999.

Paré, Denise. «Habitats, migrations et prédations. Analyse écocritique de La héronnière de

Lise Tremblay ». Cahiers de géographie du Québec 52 (2008) : 453-470.

Nora, Pierre, dir. Les lieux de mémoire. Tome 1 «La République ». Paris : Gallimard, coll.

« Bibliothèque illustrée des Histoires », 1984.

Serres, Michel. Biogée. Paris : Le Pommier, 2013 [2010].

Tansi, Sony Labou. L'Anté-peuple. Paris : Seuil, 1983.

\begin{abstract}
Notes
${ }^{1}$ L'essayiste Belinda Cannone emploie le terme de «hors-soi » pour désigner ce mouvement de l'esprit vers l'extérieur. C'est une façon d'être au monde qui désigne une attitude poétique, un mouvement de la pensée qui invite à prendre conscience du monde environnant. Il est question de traverser le monde en le regardant. Non pas seulement d'y poser les yeux, mais de s'extraire de son individualité. L'hors-soi est alors ce qui rend le réel accessible à nos yeux et décentre notre être de notre intériorité. C'est une ouverture à l'altérité qui conduit à s'ouvrir au monde et à demeurer dans le présent.

${ }^{2}$ Le fang est un dialecte bantou et désigne une communauté ethnique qui fait partie du groupe des «Pahouin» auquel appartient Ludovic Obiang. On retrouve les locuteurs de cette langue dans la région du bassin du Congo. $\mathrm{Au}$ Gabon, ceux et celles que l'on désigne sous le terme «fang » ont en partage la même histoire, langue, identité et culture.

${ }^{3}$ Le concept de «lieu de mémoire », théorisé par l'historien Pierre Nora, met en avant les dimensions fonctionnelle, symbolique, monumentale et topographique d'un lieu. Ce lien entretenu entre la mémoire et l'espace, Nora l'identifie en tant que « microdiscours du passé » pour faire allusion à cette dimension historique de la spatialité. En réactivant des valeurs communes, certains topoï deviennent des repères où s'actualisent des moments oubliés. C'est dans ce sens qu'il procède à «un inventaire des lieux où elle s'est électivement incarnée » (30). Musées, bibliothèques nationales, monuments historiques sont à ce titre dotés d'une dimension historique dans laquelle l'histoire d'une société s'inscrit. Les lieux du monde se voient donc interconnectés à l'histoire de ceux qui la peuplent car sur ces lieux, des marques qui présentifient le passé se greffent. En raison de cette dimension mémorielle et symbolique de certains lieux, on associe de plus en plus l'identité d'une société à celle de ses lieux qui participent à fonder son identité et de visualiser « ce qui a été ».

${ }^{4}$ Le concept de reterritorialisation apparaît dans l'ouvrage L'Anti-CEdipe en 1972 sous la plume des philosophes Gilles Deleuze et Félix Guattari. Ce concept de reterritorialisation, inséparable de son corollaire déterritorialisation, constitue une philosophie de l'espace. En partant de l'hypothèse que le territoire est une zone d'inscription spatiale où s'enlise le chaos et s'organise la pensée, la déterritorialisation devient la perte de cet espace d'identification; la reterritorialisation s'envisage quant à elle comme la possibilité d'enraciner sa pensée dans un espace physique ou non. Dès lors, si se déterritorialiser évoque la rupture à une potentielle aliénation, la reterritorialisation devient cette possibilité de s'identifier à nouveau . En écocritique, la reterritorialisation fait écho à une reconnexion à la terre. Il ne s'agit pas dans ce cadre de revenir à un éden perdu, mais plutôt de trouver un espace sur lequel on aura la possibilité de définir son identité, d'implanter ses racines.

${ }^{5} \mathrm{La}$ «biogée » est un terme de Michel Serres qui désigne le monde dans lequel nous évoluons avec les mots «bio» pour «vie» et «gé » pour «la Terre». Dans le livre éponyme, il donne successivement la voix aux différents éléments naturels (mer et fleuve, terre et monts, trois volcans, vents et météores, faune et flore) car, selon lui, « [n]otre Terre parle... elle annonce que le mur se fissure et va s'effondrer » (12).

${ }^{6}$ Le «vivre-ensemble » désigne dans cette étude la capacité des êtres vivants à cohabiter ensemble. En partant de l'hypothèse que l'humain se serait éloigné de la terre, le terme de « vivre-ensemble » renvoie à un intermède, à une nature ouverte à l'altérité, sans distinctions génériques, et qui nécessite un mode d'habitation poétique par une épuration des sens.

${ }^{7}$ Dans la langue fang, « Mekè » signifie « feuilles » et « Biang » est traduit par « médicament ».
\end{abstract}


${ }^{8}$ Michel Serres s'est inscrit parmi les réformateurs d'une critique de l'environnement à la française. Il décrit dans son livre Le contrat naturel (1990) un exemple de ce que devrait être la relation entre humain et terre : une symbiose avec les éléments. Sans se montrer nostalgique de l'époque rurale, il soutient que l'homo cultivator est un modèle de conduite et de culture parce qu'il arrive à " composer avec » le non-humain. En s'appuyant sur sa capacité à s'intégrer malgré les transformations (sociales, économiques et matérielles), Serres démontre que l'agriculture peut être une piste pour «habiter» la terre. En effet, il montre comment «faire avec»les changements et crée de la sorte un contrat naturel avec la terre où l'humain est invité à habiter la terre en tant que lieu commun. 